\title{
Design and Implementation of IoT Based Remote Laboratory for Sensor Experiments
}

\author{
https://doi.org/10.3991/ijim.v14i09.13991 \\ Ramya M V ( $\left.{ }^{\square}\right)$ \\ Sri Jayachamarajendra College of Engineering, Mysuru, India \\ ramyamvesjce.ac.in \\ G K Purushothama \\ Malnad College of Engineering, Hassan, India \\ K R Prakash \\ The National Institute of Engineering, Mysuru, India
}

\begin{abstract}
This article describes the design and implementation of a remote laboratory for learning sensors-based experiments and its applications using embedded systems and Internet of Things (IoT) platform. The main objective of this remote laboratory is to enhance the learning on sensors in engineering education and dealing with the industrial automation applications. With the growing IoT platform for automation, the proposed system can monitor the sensor data and allows the learner to work from anywhere and anytime using mobile android application. Thus, learners can develop knowledge on sensors and control algorithms required for the automation industries and then deploy them on the real industrial automation modules.
\end{abstract}

Keywords - Sensor laboratory, Remote laboratory, Embedded systems, IoT, Industrial Sensors and Automation.

\section{Introduction}

Experimentation is a very useful and necessary component of learning science and technology. Traditional laboratories in the university, institutes and industrial/research centers are used for this purpose and are limited when compared to the number of aspiring learners. As developing such laboratory facilities require lot of human resources and economics, remote laboratories may provide an alternative acceptable solution to such learners who are deprived of direct laboratory access.

The concept of remote laboratory was introduced from 1999. Since then, several researchers have proposed/developed various types of remote laboratories. In 2004 a remote laboratory for automatic control was developed at the University of Siena [1].

Human efforts are reduced when things gets automated. The need for working with the traditional laboratory is reduced by automating laboratory resources [2,3]. Active learning by means of remote laboratories is especially valuable for distance education 
students and learners in the workplace [4]. An efficient complementary m-learning tool can take a whole new approach to the teaching and learning process itself [5, 6]. Further, the attempt will help in achieving a futuristic model of laboratory using IoT.

An embedded system plays an important role in IoT due to their unique features, such as real time computing, and hence it is increasingly used as an effective platform for Industrial automation [7]. This provides the best communication and computing platform for industrial machineries and sensors. In industrial automation, proximity sensors [8] play an important role in various applications such as position control, conveyor system control, process control, robotic welding and machine control etc.

There are several types of proximity sensors such as inductive sensor, capacitive sensor, magnetic sensor and light sensor etc. For illustration purpose, experiments using inductive sensor is described. This work is to design and implement a remote laboratory to study the working of the proximity inductive sensors [9] and its applications in industrial automation [10].

The paper is divided into five sections. In the remaining part, Sections 2 and 3describe the system architecture and the details of Implementation, respectively. Section 4 provides the results of the remote laboratory experiments and conclusions are drawn in Section 5.

\section{System Architecture}

The basic requirements for the implementation of a remote laboratory are physical laboratory, embedded system and IoT platform. The general architecture can be represented as shown in Fig.1.

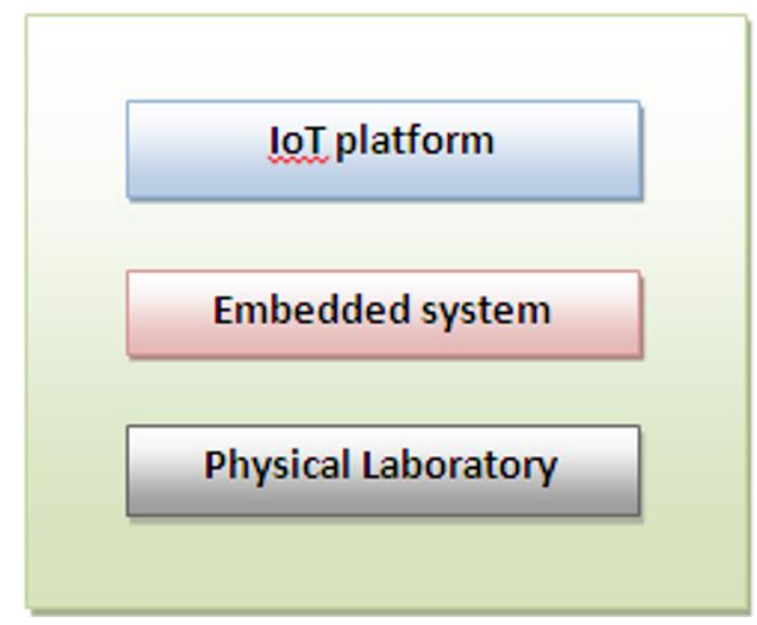

Fig. 1. General block diagram of remote laboratory 


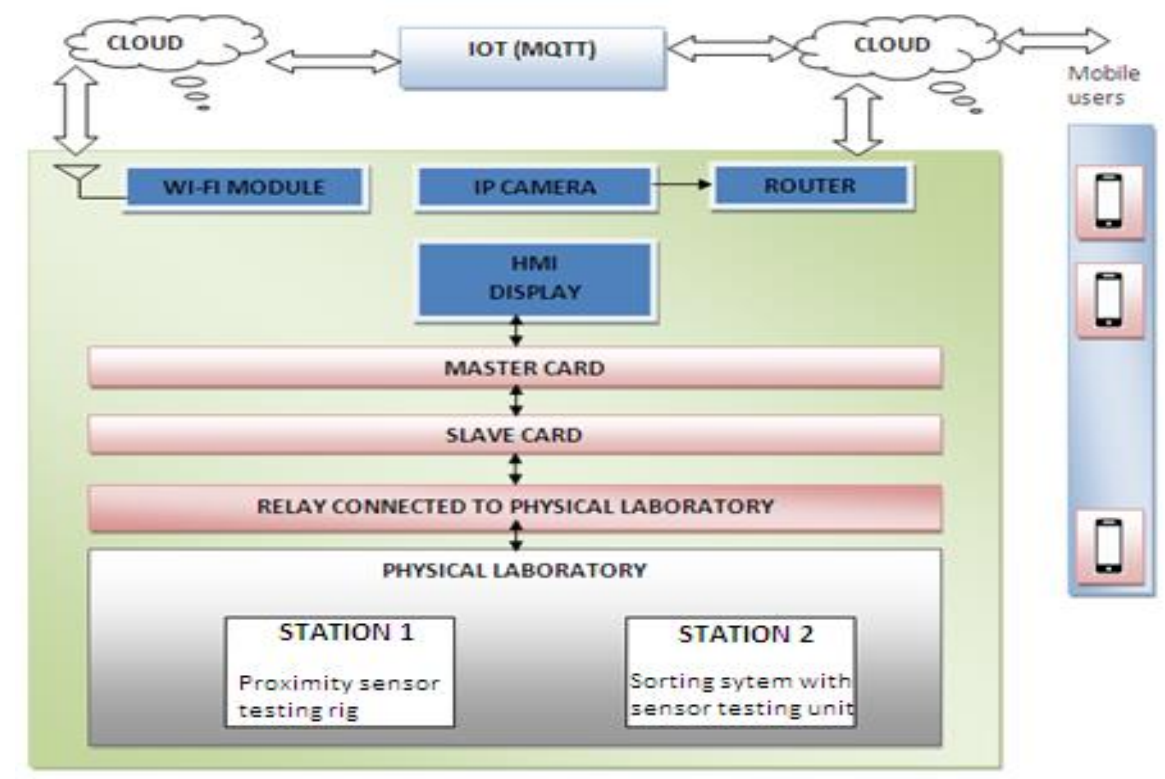

Fig. 2. Block diagram of IoT based sensor laboratory

For the present work, the selected architecture for the implementation of sensor laboratory is shown in Fig.2. The system is capable of communicating with android mobile application of learner from anywhere and at any time. The details of the implementation are described below.

\subsection{Physical laboratory}

The Physical laboratory consists of experimental setup to study the working of sensors and applications of the sensors in industrial automation. The experimental stations are as follows:

- Station 1 consists of a designed testing rig, to study the different types of proximity sensors. For illustration purpose proximity inductive sensor is considered.

- Station 2 consists of application setup for using sensors in industrial automation. For illustration purpose sorting system with sensor testing unit is considered.

\subsection{Embedded system}

The embedded system mainly consists of a master card, a slave card and a relay card used to connect between microcontroller and physical laboratory.

- Master card: ARM STM-32 microcontroller is used as Master Card. With the available 3 number of UARTs in Master card, communications is established among Slave card, Wi-Fi module and HMI display respectively. 
- Slave card: ARM STM-32 microcontroller is used as Slave card. A block of relays are used to connect between slave card and physical experimental setup to control the experimental setup.

- $\mathbf{I}^{2} \mathrm{C}$ communication protocol: $\mathrm{I}^{2} \mathrm{C}$ communication protocol is used between EEPROM and master card to store device password, Wi-Fi address, server address and port number.

- HMI display: HMI display gives the status of input and output of the experimental setup to the learner. Using HMI touch panel, inputs can be given and outputs can be observed at physical laboratory.

\subsection{IoT platform}

Message Queuing Telemetry Transport protocol (MQTT) is an IoT connectivity protocol. The IoT platform consists of following modules:

- Wi-Fi module: ESP12E Wi-Fi module is used to provide SSID (service set identifier) and network key. With the MQTT protocol, the learner can select the experiment with the help of android mobile application.

- IP camera and router: The RTSP (Real Time Streaming Protocol) is used by IP camera and the Router to send audio or video signals live from physical laboratory to remote user with mobile android application.

- Android Remote Lab (RL) mobile application : An Android mobile application has been developed for this learning purpose and it is provided to the users (learners). The learner can connect to the physical laboratory with the help of User Interface (UI) Android mobile application.

\section{Experimental Setup}

The Implementation of the experimental setup is taken in two stations: Station 1 and Station 2.

\subsection{Station 1- Proximity inductive sensor}

The objective of this station is to design and develop the working of proximity sensor testing rig. Various types of proximity sensors are used for detecting the presence or absence of an object. An Inductive proximity sensor is a well-known device used in a system for detecting the presence of a metallic (conducting) target. Fig.3. shows the diagrammatic illustration of sensor testing rig for inductive proximity sensors. 


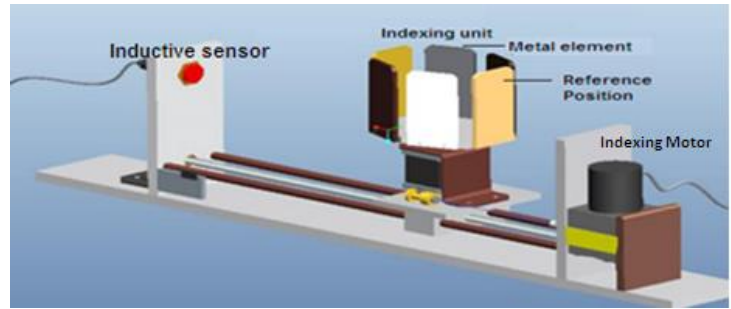

Fig. 3. Sensor testing rig

The sensor testing rig consists of inductive proximity sensors and different standard target materials like metal, rubber, glass, wood, plastic and which are all of dimension $40 \times 20 \times 1 \mathrm{~mm}$. These target materials are preloaded in the indexing unit and it is controlled by indexing motor (IM).

By turning ON the switch using android RL mobile application by the distant learner, the signal is detected by the IoT-embedded system and the preloaded program will be executed. A signal will then excite the IM which will start and comes to the reference point near the proximity inductive sensor. After reaching reference position the indexing unit will rotate for one revolution or till the target is detected as metal. Once metal target element is detected in the indexing unit, the indexing unit stop rotating and the same signal will be sent to the mobile application screen. In case, there is no metal target found in the indexing unit, the indexing unit will complete one revolution and stops.

\subsection{Station 2 - Sorting of metal and non-metal elements for industrial application}

The objective of this station is to understand the working of sorting systems in industries. The automated sorting systems can speed up the production process, gives higher throughput rates. Sorting of metal and non-metal process consists of a testing unit which is equipped with inductive, light and capacitive sensors as shown in Fig. 4.

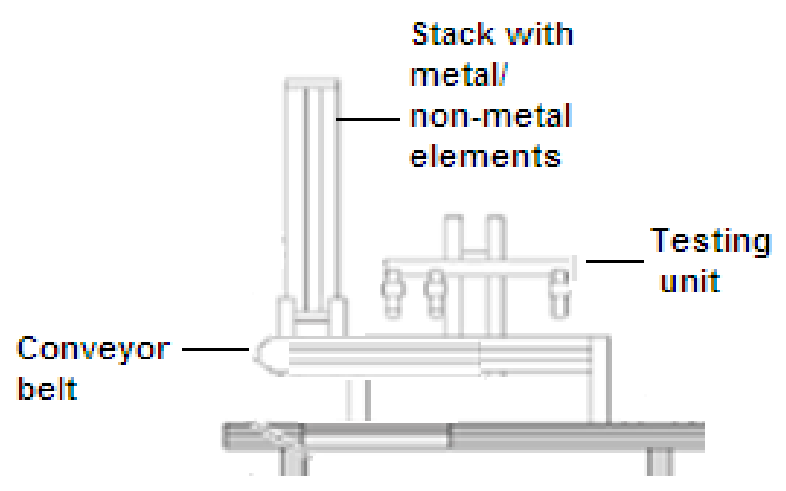

Fig. 4. Sorting system for industrial application 


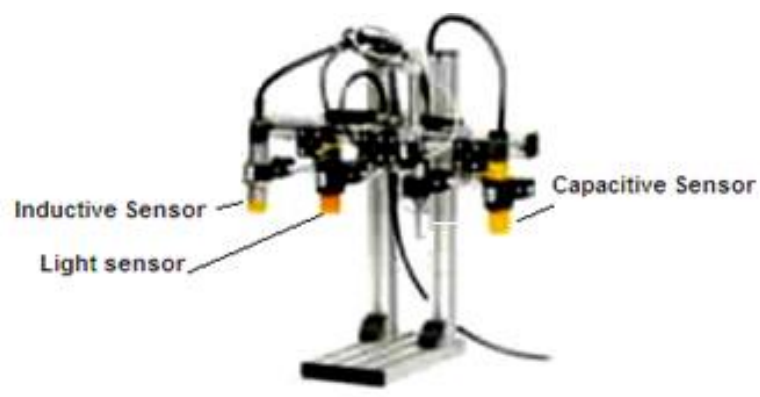

Fig. 5. Testing unit with different sensors

By turning ON the input switch, the conveyor motor start moving with one test element, that is pushed from stack magazine (which is preloaded with test materials), which results in the object to move on the conveyor. When a test material (metal or non-metal element) moves near to the capacitive sensor, it detects the arrival of the material. The separation of metal and non-metal element is then carried out by the inductive sensor. The Inductive sensors detect only the conductive materials and it works on the principle of electromagnetic induction. Fig. 5 shows the testing unit with different sensors used in the sorting system.

If the test material is detected as metal by the inductive sensor, the conveyor is moved in forward direction; the material is picked and dumped in a Bin1. If the test material is non-metal, the conveyor is moved in backward direction, and the material is picked and dumped in Bin2. The process is repeated till all the test materials are sorted.

\section{$4 \quad$ Results and Discussion}

Embedded system with IoT based kit for sensor laboratory is as shown in Fig. 6. The input lines (from sensors or switches) and output lines (to motors or relays) are connected to slave card. The slave card transmits these signals to the master card. The master card processes the data and communicates to the IoT and slave card. The details of the experimental results are discussed below. 


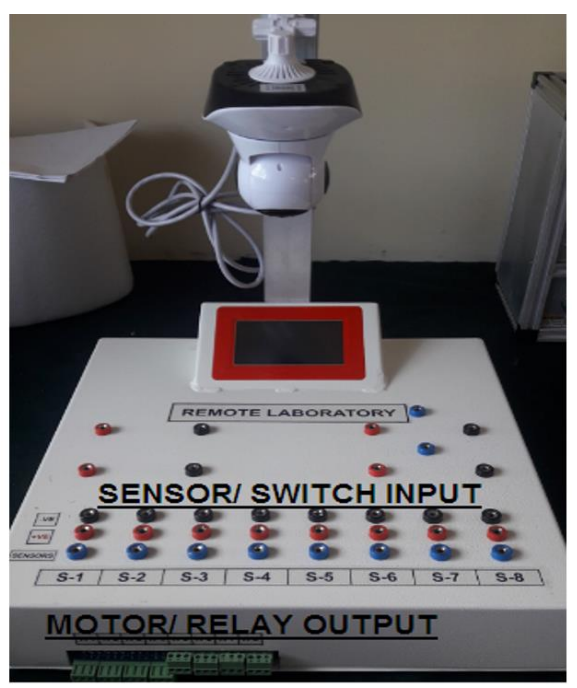

Fig. 6. Embedded system with IoT kit for sensor laboratory

\subsection{Station 1- Proximity sensor testing rig}

When the input (starting signal) is given to the switch through RL mobile application by the learner, the indexing unit will come to the reference position with the help of index motor connected to the kit. The Index unit starts rotating from reference position and when the inductive sensor detects the metal element, the motor stops rotating. The stopping of the indexing unit before completing one rotation indicates the detection that the test material is a metal. This will be indicated to the learner in their RL mobile application. Fig. 7 shows the implementation of proximity sensor testing rig.

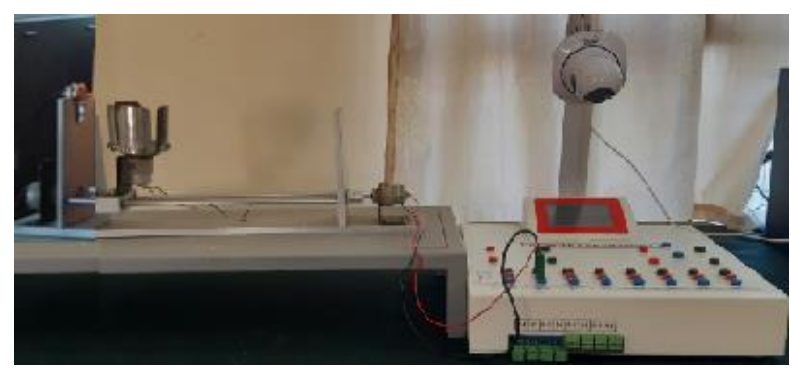

Fig. 7. Implementation of Station 1- Proximity Sensor testing rig

From Fig. 8, when the input is given to the switch IRL1=ON (RED) through mobile application, the index motor output ORL1=ON (RED) and indexing unit will come to the reference position. 

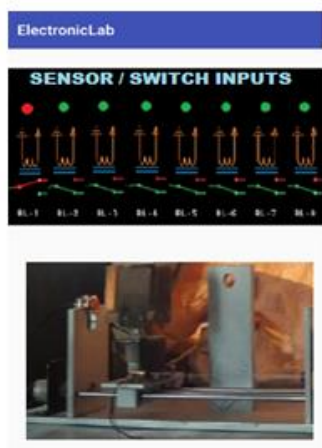

Input IRL1 - Start switch ON (RED)
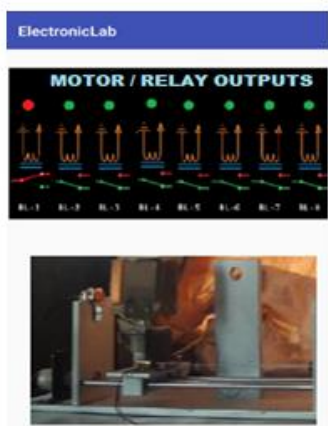

Output ORL1 - Index motor ON (RED) reference point

Fig. 8. Output of Station 1- Proximity Sensor working in mobile application

Index motorORL1=ON (RED) starts rotating from reference position along with indexing unit and when the inductive sensor detects the metal element in the indexing unit in the kit IRL2=ON (RED) and Index motor stops rotating ORL1=OFF (GREEN) as shown in Fig.9, indicating the detection of metal.
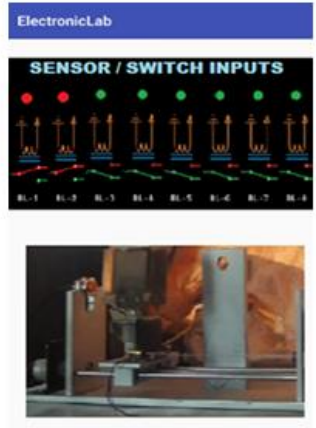

Input IRL1 - Start switch ON (RED)

Input IRL2 - Sensor ON (RED) detection of metal element
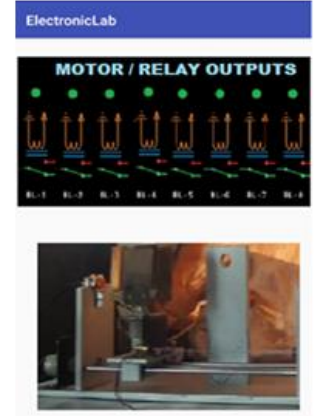

Output ORL1 - Index motor OFF (GREEN)

Fig. 9. Output of Station 1- When Proximity Inductive sensor detects Metal element in Indexing unit

The work station can be used for the demonstration of other types of proximity sensors in a similar way.

\subsection{Station 2 - Sorting of metal and non-metal elements for industrial application}

The station 2 is developed for sorting of metal and non-metal elements. When the input (start signal) is given to the kit shown in Fig. 10, one test material is pushed from 
the stack magazine onto the conveyor belt. The conveyor belt moves it past the testing unit which has two sensors: capacitive and inductive. The capacitive sensor will detect the arrival of a test material (whether metal or non-metal), and the corresponding indicator in the RL mobile application is turned ON. If the test material is a metal, the inductive sensor detects it and the corresponding indicator is $\mathrm{ON}$, the conveyor belt moves in the forward direction and stops. After checking the inductive sensor indicator, the test material is picked and dumped a Bin1. The stack magazine will then push the second test material and the process is repeated. If the test material is non-metal the inductive sensor indicator is checked and as it is now OFF, the conveyor is moved in the reverse direction and the test material is now picked up and dumped in Bin2. The next test material is then taken from the stack magazine and process is repeated till all the test materials are sorted.

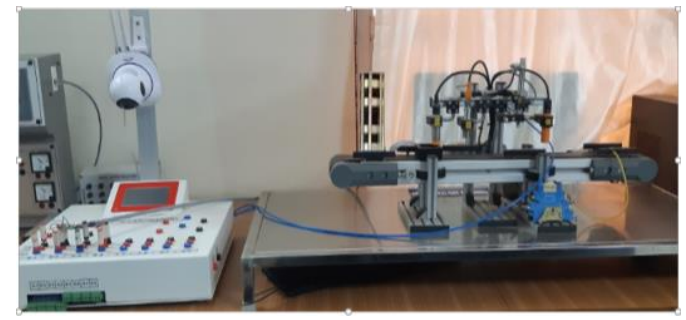

Fig. 10.Station 2 - Sorting of metal and non-metal elements for industrial application
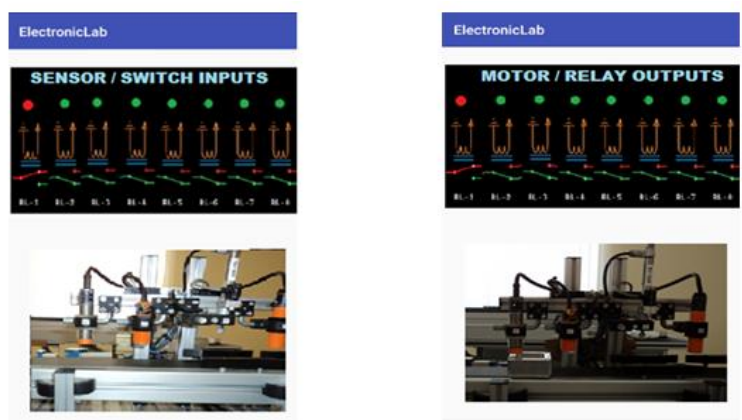

$$
\begin{aligned}
& \text { Input IRL1 - Start switch ON (RED) one cube } \\
& \text { pushed on conveyor }
\end{aligned}
$$

pushed on conveyor

$$
\begin{aligned}
& \text { Output ORL1 - Conveyor motor ON (RED) } \\
& \text { along with cube starts moving in } \\
& \text { forward direction }
\end{aligned}
$$

Fig. 11.Station 2 - Implementation of Sorting of metal and non-metal elements for industrial application

When the input switch IRL1= ON (RED) a metal or non-metal test material is pushed from the stored stack magazine and output ORL1= ON (RED)conveyor motor turned on, starts running the conveyor in forward direction as shown in Fig. 11.

Fig.12. shows when the metal element passing on conveyor, the inductive sensor IRL2 $=$ ON (RED) and the conveyor will move in forward direction ORL1= ON (RED) and stops to collect in Bin1. 

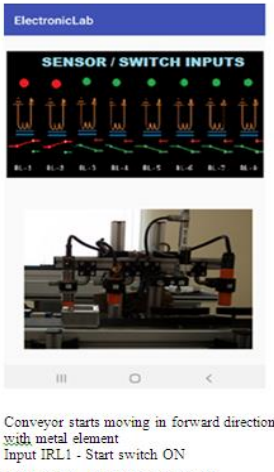

nput IRL2 - Inductive sensor O

Input IRL3 - Capacitive sensor OFF (Material already passed on conveyor)
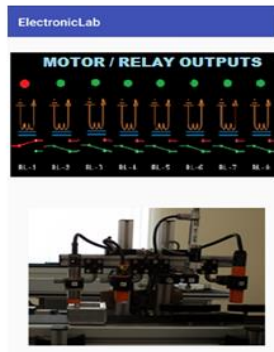

Ouput ORL $1=O N$ Conveyor motor ON starts moving in forward direction
whentRL $2=0 \mathrm{~N}$

Fig. 12. Station 2 - Implementation of Sorting of metal element output
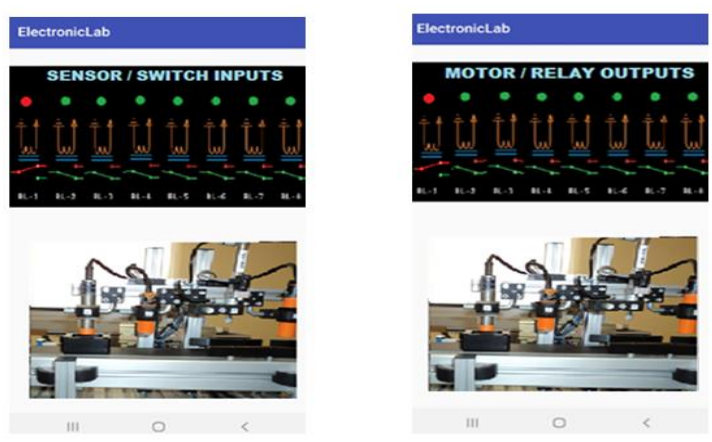
Conveyor with non- metal element
Input $1 R L 1$ - Start switch ON
Input $1 R L 2$ - Inductive sensor OFF
Input IRL3 - Capacitive sensor OFF

Output ORL $1=$ ON Conveyor motor ON
starts moving in reverse direction when

Fig. 13.Station 2 - Implementation of Sorting of non- metal element

Fig. 13 shows, when the non-metal element passing on conveyor, the inductive sensor IRL2 = OFF (GREEN) and the conveyor ORL1= ON (RED) reaches the end, and then conveyor goes in reverse direction and stops, the material is then picked in Bin2. Hence the sorting of metal and non-metals is carried out.

\section{Conclusion}

The work presented in this article describes the concept of IoT based remote laboratory for sensor experiments. The sensor module is used for monitoring and controlling the various parameters of industrial automation. The experimental results reported are encouraging. Such a training and knowledge may lead design and development of more and more innovative automation systems. 
It is necessary to note that remote laboratories are not created to substitute the physical laboratory, but to complement them. These will allow the learners to conduct experiments remotely while still perceiving the actual laboratory work.

\section{References}

[1] M. Casini, D. Prattichizzo, and A. Vicino, - The automatic control telelab, IEEE Control Syst. Mag., vol. 24, no. 3, PP: 36-44, Jun- 2004

[2] Coia Ferrater-Simon, Lluis Molas-Balada, Oriol Gomis-Bellumunt, "A Remote Laboratory Platform for Electrical Drives Control Using PLC”, IEEE Trans. Educ., Vol.52, PP: 425435, Aug-2009. https://doi.org/10.1109/te.2008.930095

[3] Luis Gomes, Seta Bogosyan, "Current Trends in Remote Laboratories", IEEE Trens, Industrial Electronics, Vol, No.12, PP: 4744-4755, Dec-2009. https://doi.org/10.1109/ $\underline{\text { tie. } 2009.2033293}$

[4] D. Ursutiu, M. Ghercioiu, C. Samoila and P. Cotfas, "Wi-Fi Tags for the Remote and Virtual Laboratory", iJIM: International Journal of Interactive Mobile Technologies Vol. 2, No. 2, PP:41-46, Oct- 2008

[5] R.N. Madeira, V.F. Pires, O.P. Dias and J.F. Martins "An Analog Electronics Mobile Course with a Competitive Learning Approach" iJIM: International Journal of Interactive Mobile Technologies Vol. 4, No. 4, PP:37-44, Oct- 2010. https://doi.org/10.3991/ ijim.v4i4.1415

[6] D. May, C. Terkow sky, T. Haertel and C. Pleul, "Bringing Remote Labs and Mobile Learning together", iJIM: International Journal of Interactive Mobile Technologies Vol. 7, No. 3, PP: 55-62, July- 2013. https://doi.org/10.3991/ijim.v7i3.2915

[7] Nitin Sapre, Abhishek Singh Tomar "Study of Industrial Automation with Mechatronic System Using Various Technology Embedded” ISSN (Print): 2393-8374, (Online): 2394-0697, Volume-3, Issue-7, July- 2016

[8] Hoai Nguyen "Experimental Research Dynamic Characteristic of Proximity Sensor" International Journal of Latest Engineering Research and Applications (IJLERA) ISSN: 24557137, Volume - 02, Issue - 04, PP: 49-54, April - 2017.

[9] Robert Charles, "Shielding Arrangement For sensing The Proximity of A metallic Object" International Journal of Engineering Research \& Technology (IJERT) ISSN: 2278-0181, Vol. 6 Issue 05, May - 2017

[10] Wen-Jye SHYR, "Development and Evaluation of Mechatronics Learning System in a WebBased Environment", TOJET: The Turkish online journal of education technology, Vol. 10, Issue 1, PP: 89-96, Dec-2015

\section{$7 \quad$ Authors}

Ramya M V is a research scholar in Visvesvaraya Technological University, Karnataka, India. She is working as an Assistant Professor in the Department of Electronics and Communication Engineering at Sri Jayachamarajendra College of Engineering, Karnataka, India. She has more than 8 years of experience in teaching. Her research interest is Embedded systems, IoT, Robotics and Automation. Email: ramyamv@sjce.ac.in 
Dr. G. K. Purushothama is presently serving as Professor and Head in theDepartment of Electrical and Electronics Engineering at Malnad College of Engineering, Karnataka, India. He obtained his M. E. degree in Indian Institute of Technology Madras (IIT M), India and Ph.D. degree from Indian Institute of Science (IISc), Bangalore, India. He has more than 34 years of experience in teaching. His areas of interest include Control systems, Power system optimization \& Distance learning.

Dr. K. R. Prakash is presently serving as Professor and Head, Centre for Automation Technology (CAT) at The National Institute of Engineering, Karnataka, India. He obtained his M. Tech. degree in University of Mysore, India and Ph.D. from Visvesvaraya Technological University, Karnataka, India. He has more than 26 years of experience in industry and teaching. His areas of interest include Control Systems, Automation \& Robotics.

Article submitted 2020-01-11. Resubmitted 2020-03-12. Final acceptance 2020-03-14. Final version published as submitted by the authors. 